\title{
Initial-link duration and acquisition of preference in concurrent chains
}

\author{
MARK E. BERG and RANDOLPH C. GRACE \\ University of Canterbury, Christchurch, New Zealand
}

\begin{abstract}
Initial-link response allocation in concurrent chains becomes less extreme as the absolute duration of the initial links increases (Fantino, 1969). The present study asked whether initial-link duration affected how quickly response allocation reached asymptote (i.e., acquisition of preference). Six pigeons were trained on a concurrent-chains procedure in which the terminal links were fixed-interval (FI) $8 \mathrm{sec}$ FI $16 \mathrm{sec}$ or FI $16 \mathrm{sec}$ FI $8 \mathrm{sec}$ and were reversed every 20 sessions. Across conditions, all possible combinations of transitions between variable-interval (VI) 8-sec (short) and VI 24-sec (long) initial-link schedules were studied. Overall, the rate of acquisition was faster when the durations of the initial links preceding the reversal were short rather than long, and when the durations of the initial links following the reversal were long rather than short. By contrast, initial-link duration had no effect on acquisition or asymptotic measures of temporal control of terminal-link responding. These results support the core principle of delay-reduction theory (Fantino, 1969) that the impact of a conditioned reinforcer varies directly with initial-link duration, but also suggest that temporal learning during the terminal links proceeds independently of initial-link duration.
\end{abstract}

Concurrent-chains procedures are widely used in the study of behavioral choice and allow researchers to investigate the dynamics of response allocation in complex situations. In a typical version of the procedure with pigeons, two side keys in an experimental chamber are illuminated white to signal the choice phase or initial links. Responses to the side keys during the initial links occasionally produce access to one of two terminal-link schedules. If a left initial-link response produces a terminal link, the center key is illuminated red; for a right-key response, the center key is lighted green. Responding during the terminal links is reinforced with access to food, after which the initial links are reinstated and the next cycle begins.

The terminal-link stimuli have often been viewed as conditioned reinforcers, because they are paired with primary reinforcement and their presentation maintains behavior during the initial links. Thus, initial-link response allocation provides a measure of the relative strength or value of the terminal-link stimuli as conditioned reinforcers (Grace \& Savastano, 2000). The typical result is that pigeons will respond more to the initial link leading to the terminal link associated with the shorter delay to reinforcement. For example, if the schedules associated with the red or green center key are fixed-interval (FI) $8 \mathrm{sec}$ and FI 16 sec, respectively, response allocation will favor the left initial link, so the red center key is said to be relatively more effective as a conditioned reinforcer.

These data were presented at the annual meeting of the Association for Behavior Analysis, Boston, May 2004. Address correspondence and reprint requests to M. E. Berg or R. C. Grace, University of Canterbury, Department of Psychology, Private Bag 4800, Christchurch, New Zealand (e-mail: bmark3@xtra.co.nz; randolph.grace@canterbury.ac.nz).
Many studies in concurrent chains have utilized designs in which subjects are given extensive experience with a set of schedules - often 20 or more sessions - to allow behavior to stabilize before conditions are changed. Results from these studies can be accurately described by various quantitative models, including Grace's $(1994,1996)$ contextual choice model (CCM) and Mazur's (2001) hyperbolic-value added model (HVA). Both models are based on the generalized matching law (Baum, 1974) and make the assumption that the primary determiner of initial-link responding is the relative value of the terminal-link schedules. These models are typically able to account for $90 \%$ or more of the variance in relative initial-link responding, providing a quantitative description of response allocation in concurrent chains comparable to that of the generalized matching law for concurrent schedules (Baum, 1979).

Although much is known about the determiners of steady-state behavior in concurrent chains, researchers have recently begun to study acquisition - how preference changes when the contingencies are altered. For example, Mazur, Blake, and McManus (2001) compared the rate of change of initial-link response allocation depending on whether the initial- or terminal-link schedules were changed. In one set of conditions, both terminal-link reinforcement delays were $12.5 \mathrm{sec}$ while the initial-link key delivering the higher percentage of terminal-link entries (80\% vs. $20 \%$ ) was changed. In other conditions, both initial links delivered $50 \%$ of the terminal-link entries, but the terminal link associated with the shorter delay to reinforcement was changed ( 5 vs. $20 \mathrm{sec}$, or $2 \mathrm{vs} .18 \mathrm{sec}$ ). Mazur et al. found that preference adjusted more rapidly after changes in the percentage of terminal links delivered by the initial links rather than when the terminal-link delays were switched. Mazur (2002) investigated whether 
rate of change in preference was affected by terminal-link duration. In his experiment, the terminal-link delays were equal ( 1 or $20 \mathrm{sec}$ ), and the percentage of terminal-link entries provided by the initial links was changed across conditions. Overall, Mazur (2002) found no effect of terminal-link duration on acquisition. Although initiallink response rates were much higher when the terminallink delays were $1 \mathrm{sec}$, the rate of change in preference was the same as in the 20 -sec condition.

Grace (2002a, Experiment 1) studied acquisition of preference in concurrent-chains using a successivereversal design in which the location of the richer terminallink schedule was changed every 20 sessions. The terminallink schedules were either both FI or both VI, and the overall durations were short ( 8 vs. $16 \mathrm{sec}$ ) or long (16 vs. $32 \mathrm{sec})$. Across conditions, he studied all possible combinations of terminal-link schedule pairs both preceding and following the reversal. Results supported the following generalization: Terminal-link schedule pairs that were relatively high in value (e.g., VI rather than FI, short rather than long) produced faster rates of change when they followed the reversal, but also greater interference (i.e., slower rates of change) when they preceded the reversal. Grace showed that the results were consistent with predictions of a simple linear-operator model (LINOP), which also provided a good quantitative description of the difference in acquisition rates across the conditions.

There is an apparent conflict in the results of Mazur (2002) and Grace (2002a), because Mazur found that acquisition rate did not depend on overall terminal-link duration, whereas Grace found that preference changed more rapidly when the durations were long preceding and short following the reversal. The reason for the apparent discrepancy is that Grace (2002a) used a design in which the schedules preceding and following the reversal were manipulated factorially, so that their effects could be assessed independently. According to the LINOP model, when the durations preceding and following the transition are the same, as in Mazur's (2002) study, their effects cancel out. Thus, the LINOP model is able to account for the results in both Grace's and Mazur's studies.

The purpose of the present research was to determine whether rate of acquisition was affected by initial-link duration. The effect of this variable on steady-state preference is well known. In a classic experiment, Fantino (1969) showed that preference for the richer terminal link (VI $30 \mathrm{sec}$ ) decreased toward indifference as the duration of the equal initial-link schedules increased from VI 30 to VI 600 sec, a result now known as the "initial-link effect." Our goal was to address the analogous question for acquisition. Given a constant pair of terminal-link schedules, will the rate of change in preference when the schedules are reversed depend on the overall duration of the initial links? We planned to assess the effects of initial-link duration both preceding and following the schedule change independently, by using a factorial design similar to that of Grace (2002a).
According to the LINOP model (Grace, 2002a), rate of acquisition depends on the combined value of the terminal-link schedules both preceding and following the schedule reversal. Because terminal-link value is determined by the delays to reinforcement signaled by the terminal-link stimuli, the LINOP model predicts that the rate of acquisition should be the same regardless of initiallink duration. However, a study by Mazur (1997) suggests a different result. He found that individual reinforcers had greater impact on change in preference in concurrent VI VI schedules when the overall reinforcement rate was low rather than high. In Mazur's (1997) experiment, the overall reinforcement rate was the same both preceding and following the transition, which consisted of a switch in relative reinforcement rate from $50 \%$ to $90 \%$. If conditioned reinforcers are functionally equivalent to primary reinforcers (Berg \& Grace, 2004; Williams \& Dunn, 1991), then Mazur's (1997) results would suggest that each conditioned reinforcer (i.e., terminal-link entry) should have greater impact when the overall rate of conditioned reinforcement is low rather than high. In that case, relatively long initial-link schedules prior to terminal-link reversal should generate more interference, and hence retard acquisition, whereas if initial-link schedules are relatively long following the reversal, each terminal link should have greater impact, increasing the rate of acquisition. Thus, we would expect preference to change most rapidly, relative to the eventual asymptotic value, when the initial links were short preceding the reversal and long following the reversal; to change least rapidly for the reverse (i.e., long preceding and short following); and for the rate of change to be intermediate when the initial links preceding and following the reversal were either both short or both long.

In our experiment, pigeons responded in a concurrentchains procedure in which the terminal links were FI $8 \mathrm{sec}$ and FI $16 \mathrm{sec}$. A successive-reversal design similar to that of Grace (2002a) was employed, in which the schedules were switched every 20 sessions. In different conditions, the initial-link schedule was either VI $8 \mathrm{sec}$ (short) or VI $24 \mathrm{sec}$ (long), and across conditions, all possible combinations of initial-link durations preceding and following the reversal were explored (i.e., short-short, short-long, longlong, long-short). In addition, we used a procedure introduced by Grace and Nevin (1999; see also Grace, 2002b), where $25 \%$ of terminal links were "no food" trials on which the stimulus was presented for $48 \mathrm{sec}$ and no reinforcement was delivered. Thus, the terminal links were analogous to trials in the peak procedure (Roberts, 1981), allowing us to observe the temporal control of terminal-link responding concurrently with initial-link preference.

\section{METHOD}

\section{Subjects}

Six pigeons numbered 191, 193, 194, 196, 197, and 198 participated as subjects and were maintained at $85 \%$ of free-feeding weight $\pm 15 \mathrm{~g}$ by postsession feedings. They were housed individually and were allowed free access to water and grit, in a vivarium with a 
12:12-h light:dark cycle (lights on at $0700 \mathrm{~h}$ ). All had experience with a variety of experimental procedures.

\begin{abstract}
Apparatus
Six standard three-key operant chambers, $350 \mathrm{~mm}$ deep $\times$ $360 \mathrm{~mm}$ wide $\times 350 \mathrm{~mm}$ high, were used. The keys were $260 \mathrm{~mm}$ above the floor, arranged in a row. In each chamber, a houselight was located $70 \mathrm{~mm}$ above the center key, and a grain magazine with an aperture $(60 \times 50 \mathrm{~mm})$ was $130 \mathrm{~mm}$ below the center key. The magazine was illuminated when wheat was made available. A force of approximately $0.10 \mathrm{~N}$ was necessary to operate each key, and effective responses produced an audible feedback click. The chambers were enclosed in a sound-attenuating box, and ventilation and white noise were provided by an attached fan. Event scheduling and data recording were controlled with a MEDSTATE notation program and a MED-PC system interfaced with an IBM-compatible microcomputer located in an adjacent room.
\end{abstract}

\section{Procedure}

Because the subjects were experienced, training began immediately in the first condition. The houselight provided general illumination at all times except during reinforcer delivery. Sessions were scheduled 7 days per week and at approximately the same time $(1300 \mathrm{~h})$, with few exceptions.

The experiment used a version of the concurrent-chains/peak procedure introduced by Grace and Nevin (1999). Sessions ended when 72 initial-link and terminal-link cycles had been completed, or 70 min had elapsed, whichever occurred first. Cycles began with the initial links, signaled by white illumination of the left and right response keys. At the start of each cycle, either the left or the right key was selected to produce a terminal-link entry, with the requirement that out of every 8 cycles, four entries were assigned to the right key and four to the left key. Thus, the initial links used interdependent scheduling (i.e., forced choice; Stubbs \& Pliskoff, 1969). When three requirements were met, an initial-link response produced entry into a terminal link. The requirements were, first, that the response was made to the preselected key; second, that an interval sampled from the initial-link VI schedule had timed out; and third, that a 1-sec changeover delay (COD) had been satisfied (i.e., at least $1 \mathrm{sec}$ had elapsed following a changeover to the key for which the terminallink entry was arranged). The initial-link VI schedules contained 12 intervals constructed from an arithmetic progression, $a, a+d, a+$ $2 d, \ldots$, where $a$ equals $1 / 12$ and $d$ equals $1 / 6$ of the schedule value. The intervals were sampled randomly without replacement, and the initial-link schedule did not begin timing until the first response of the cycle had been made.

Terminal-link entry was signaled by simultaneously extinguishing the side keys and lighting the center key either red or green.
The color of the center key depended on whether a left or a right initial-link response had produced the terminal link (red-left, greenright). Both terminal links were presented on 36 cycles per session, of which 27 were food trials and 9 were no-food trials. On the food trials, the terminal-link schedules were always FI $8 \mathrm{sec}$ or FI $16 \mathrm{sec}$. After the schedule value had elapsed, the first center-key response was reinforced. During reinforcement, the houselight and keylights were extinguished and the grain feeder was raised and illuminated for $3 \mathrm{sec}$. A 5 -sec limited hold was in effect on the 27 food trials. If a response was not made within $5 \mathrm{sec}$ after reinforcement had been scheduled, the terminal link was canceled, the center key was extinguished, and the next cycle began. All subjects responded consistently on food trials, so that scheduled reinforcers were rarely canceled. On no-food trials, the center key was illuminated for $48 \mathrm{sec}$ and ended without reinforcement. At the end of each terminal link, the side keys were once again illuminated white, thus signaling the initial links, and the next cycle began.

The experiment consisted of a series of conditions, each lasting 20 sessions. The terminal-link schedules were switched after every condition (i.e., from FI 8 FI 16 to FI 16 FI $8 \mathrm{sec}$, or vice versa). The initial-link schedule was either VI 8 sec (short) or VI 24 sec (long) and was varied across conditions in order to exhaust all possible combinations of transitions (i.e., initial-link durations preceding and following the terminal-link reversal; short-long, short-short, long-long, and long-short). The order of conditions was counterbalanced, and it is listed for all subjects in Table 1.

\section{Measure of Peak Location}

The location of maximum response rate on no-food trials was obtained for individual sessions and subjects by using the trimmed median method of Cheng and Roberts (1991). First, a cumulative response distribution was generated for a particular session and subject, and the bin that contained the median response was located. The median time was obtained by linear interpolation. A new cumulative response distribution was then generated after excluding all responses that occurred after twice the median. A median was calculated for the new distribution, and the process was iterated until successive medians stopped changing. The final median was taken as an estimate of peak time. The advantage of this nonparametric procedure is that it provides a reliable measure of peak location from single-session data (Grace \& Nevin, 1999).

The .05 significance level was used for all statistical tests

\section{RESULTS}

The primary dependent variables were response allocation in the initial links (measured as the proportion of

Table 1

Order of Conditions for All Subjects

\begin{tabular}{|c|c|c|c|c|c|c|c|c|c|}
\hline Order & Condition & IL & TL & Condition & IL & TL & Condition & IL & $\mathrm{TL}$ \\
\hline & \multicolumn{3}{|c|}{ Bird 191} & \multicolumn{3}{|c|}{ Bird 193} & \multicolumn{3}{|c|}{ Bird 194} \\
\hline 1 & & VI 8 & FI 8,16 & & VI 24 & FI 8,16 & & VI 24 & FI 8,16 \\
\hline 2 & SS & VI 8 & FI 16,8 & LL & VI 24 & FI 16,8 & LL & VI 24 & FI 16,8 \\
\hline 3 & SL & VI 24 & FI 8,16 & LS & VI 8 & FI 8,16 & LS & VI 8 & FI 8,16 \\
\hline 4 & LL & VI 24 & FI 16,8 & SS & VI 8 & FI 16,8 & SS & VI 8 & FI 16,8 \\
\hline \multirow[t]{2}{*}{$\approx$} & LS & VI 8 & FI 8,16 & SL & VI 24 & FI 8,16 & SL & VI 24 & FI 8,16 \\
\hline & \multicolumn{3}{|c|}{ Bird 196} & \multicolumn{3}{|c|}{ Bird 197} & \multicolumn{3}{|c|}{ Bird 198} \\
\hline 1 & & VI 24 & FI 16,8 & & VI 24 & FI 16,8 & & VI 8 & FI 16,8 \\
\hline 2 & LL & VI 24 & FI 8,16 & LL & VI 24 & FI 8,16 & SS & VI 8 & FI 8,16 \\
\hline 3 & LS & VI 8 & FI 16,8 & LS & VI 8 & FI 16,8 & SL & VI 24 & FI 16,8 \\
\hline 4 & SS & VI 8 & FI 8,16 & SS & VI 8 & FI 8,16 & $\mathrm{LL}$ & VI 24 & FI 8,16 \\
\hline 5 & SL & VI 24 & FI 16,8 & SL & VI 24 & FI 16,8 & LS & VI 8 & FI 16,8 \\
\hline
\end{tabular}

Note-SS, short-short; SL, short-long; LL, long-long; LS, long-short; IL, initial-link schedule (VI 8-short; VI 24-long); TL, terminal-link schedules (left, right). 
responses to the key leading to the FI 8-sec terminal link), and the central tendency and variability of responding on no-food terminal-link trials (measured as the median and interquartile range, respectively). We also combined the medians to give a relative measure $(1 /$ PeakL $) /(1 /$ PeakL + 1/PeakR), where PeakL and PeakR are the medians (i.e., peak locations) of the response distributions during nofood trials for the left and right terminal links. Given the assumption that peak location provides an estimate of the animal's expected time to reinforcement, this measure may be interpreted as the relative expected immediacy of reinforcement (Grace, 2002b). One subject (Pigeon 198) responded nondifferentially during no-food terminal-link trials in two conditions, so that peak location and variability measures could not be computed. Terminal-link data for this subject were omitted from all analyses reported below.

Our primary purpose was to determine whether acquisition of preference was affected by initial-link duration. Group mean data across the 20 sessions of each condition are presented in Figure 1. Data shown are the relative response rate for the initial-link key associated with the FI 8-sec terminal link. Points indicated as "Base" (i.e., baseline) represent the average relative initial-link response rate across the last five sessions of the previous condition for the initial link associated with the FI $8 \mathrm{sec}$ in the current condition. Asymptotic preference (Asy) was calculated by averaging across the last five sessions in the current condition. Data for the SS and LL conditions (i.e., short-short, VI 8-sec initial links both preceding and following the reversal; and long-long, VI 24-sec initial links both preceding and following the reversal) are shown in the upper panel; corresponding data from the SL and LS conditions are shown in the lower panel.

For asymptotic responding, the upper panel of Figure 1 shows that preference for the initial link associated with the FI $8 \mathrm{sec}$ was greater in the SS than in the LL condition. By contrast, differences between the SL and LS conditions in the lower panel are less clear. A repeated measures ANOVA was performed with preceding duration, following duration, and session as factors. The main effect of session was significant $[F(19,57)=49.83]$, as
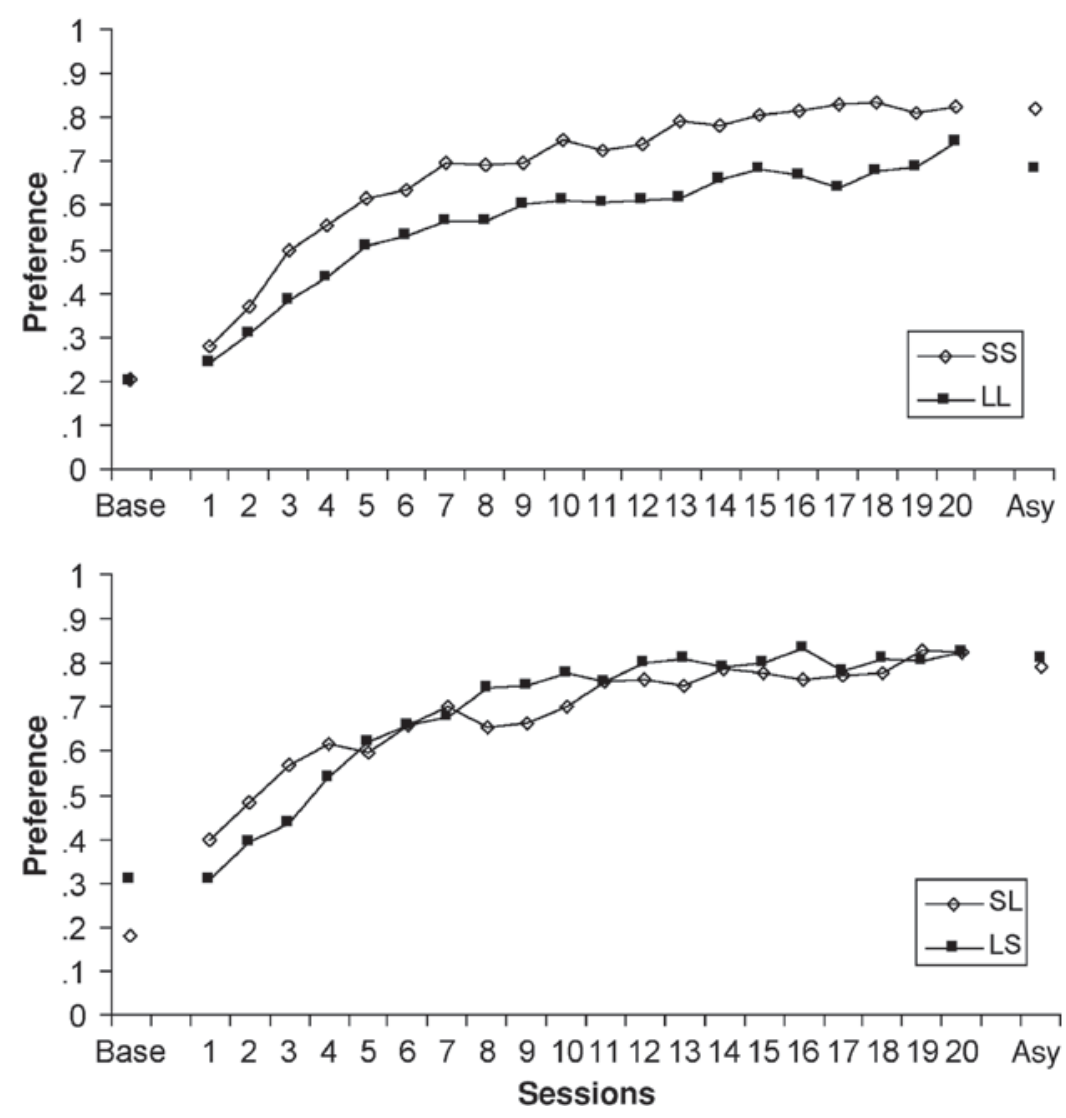

Figure 1. Average preference, measured as the proportion of responses to the initiallink preceding the FI 8-sec terminal link, plotted across the 20 sessions of each condition. Points labeled "Base" represent the average relative initial-link response rate across the last 5 sessions of the previous condition. Points labeled "Asy" are the average of the last 5 sessions in the current condition. The unfilled diamonds in the top panel represent the short-short (SS) initial-link transition, and filled squares, the long-long (LL) transition. In the bottom panel, the unfilled diamonds indicate the short-long (SL) transition and filled squares the long-short (LS) transition. 
was the interaction between following duration and session $[F(19,57)=2.99]$. The main effect and interactions for preceding duration were not significant. The following duration $\times$ session interaction was due to a crossover such that early in the condition, preference for the FI 8-sec terminal link was more extreme when the following duration was long, whereas toward the end of the condition, preference for the FI $8 \mathrm{sec}$ was more extreme when the following duration was short. A planned contrast found that asymptotic preference for the FI $8 \mathrm{sec}$ was significantly greater when the following duration was short $(M=0.82)$ than when the following duration was long $(M=0.74)$ $[F(1,5)=6.73]$. This confirms that the "initial-link effect" was obtained (Fantino, 1969).

Figure 2 displays the data for terminal-link relative expected immediacy in the same format as in Figure 1. Across conditions, no systematic differences were apparent. A repeated measures ANOVA was performed on these data with preceding duration, following duration, and session as factors. The results were consistent with a visual inspection of Figure 2, indicating a significant effect of session $[F(19,79)=7.17]$, but no significant effects of preceding or following duration and no significant interactions. Averaged across subjects and conditions, the asymptotic relative expected immediacy was 0.65 , which is close to the programmed relative immediacy $(0.67)$.
One difficulty with analyzing acquisition in terms of relative initial-link response rates, such as the data in Figure 1 , is that both the extent and rate of change may differ across conditions. To control for differences in extent of change, data were normalized as follows. The change in each session, relative to baseline, was expressed as a proportion of the eventual change, $\left(x_{i}-x_{n}\right) /\left(x_{n+1}-x_{n}\right)$, where $x_{n}$ and $x_{n+1}$ are asymptotic preference for conditions $n$ and $n+1$, and $x_{i}$ is preference for the $i$ th postreversal session in condition $n+1$. In this way, data from all conditions are placed on a common scale, where 0 indicates no change from previous baseline and 1 means that asymptotic performance has been reached.

Normalized preference data from all conditions, averaged across subjects, are shown in Figure 3. Individual data were entered into a repeated measures ANOVA with the duration of the initial links preceding and following the reversal and session as factors. Only data from the first three sessions were used for this analysis, because the largest potential differences in acquisition rate across conditions are observed during the early postreversal sessions (Grace, 2002a). The effect of preceding duration was significant $[F(1,3)=18.06]$, indicating that acquisition was faster when the initial links preceding the reversal were short rather than long. The effect of following duration was also significant $[F(1,3)=7.99]$, showing
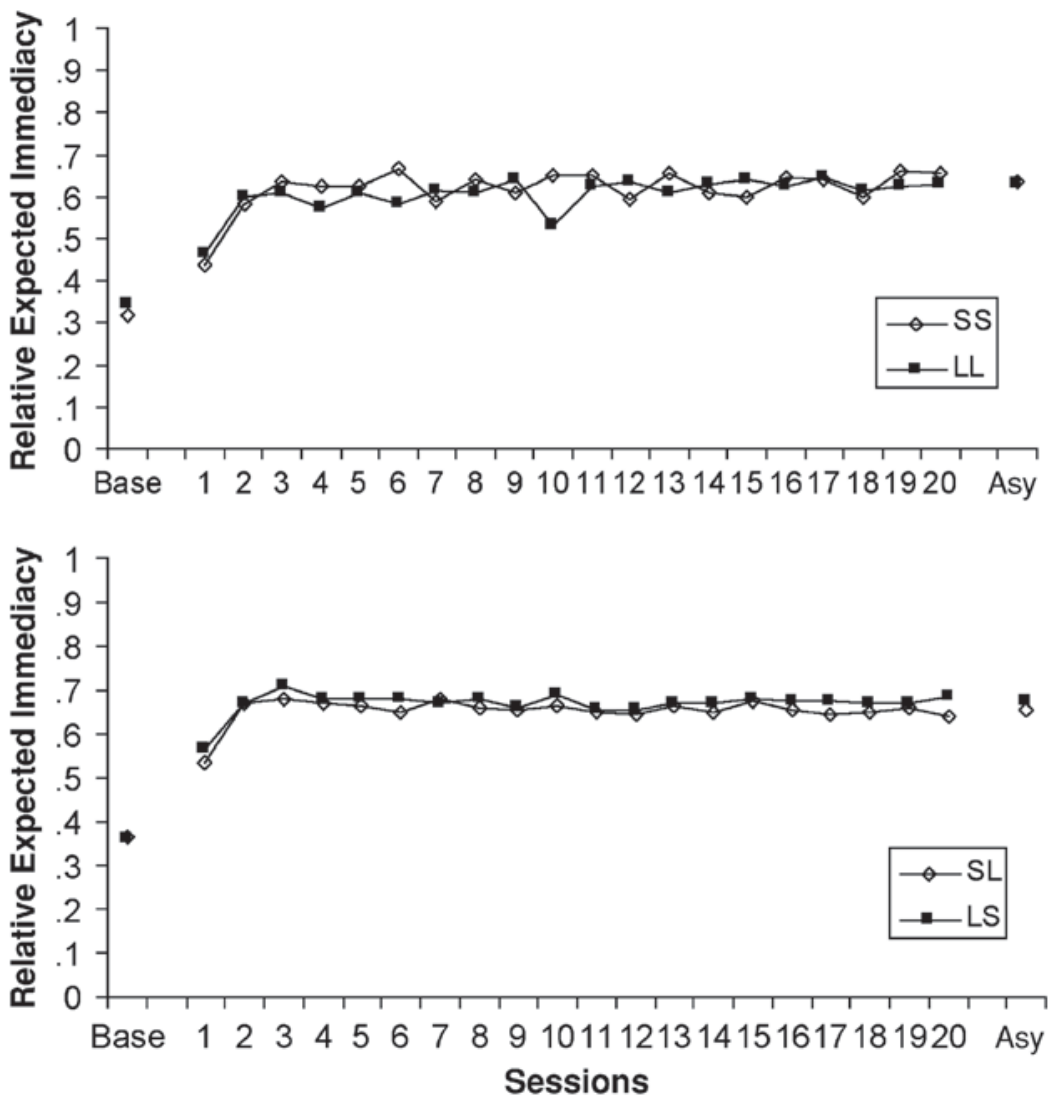

Figure 2. Average relative expected immediacy, plotted across the 20 sessions of each condition. The legend and other labels are the same as in Figure 1. 

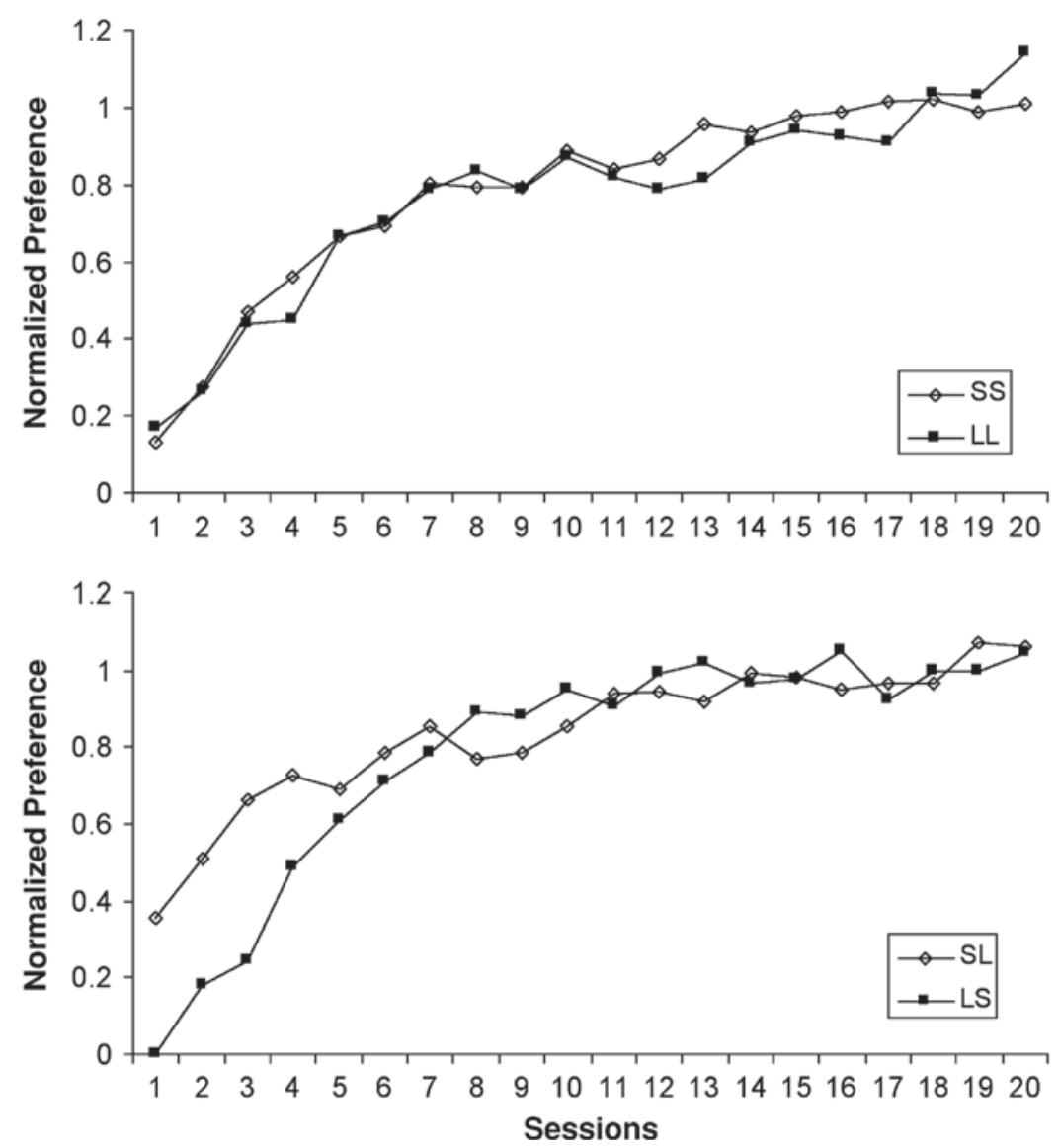

Figure 3. Average normalized preference values plotted across the 20 sessions of each condition.

that acquisition was faster when the initial links following the reversal were long rather than short. As expected, the effect of session was significant $[F(2,11)=85.43]$, but none of the interactions were. Averaged across sessions and subjects, the normalized preference achieved during the first three sessions was 0.51 for short-long $(S E=.04)$, 0.30 for short-short $(S E=.05), 0.26$ for long-long $(S E=$ $.05)$, and 0.15 for long-short $(S E=.07)$. Thus, acquisition was fastest for the short-long condition, slowest for the long-short condition, and intermediate for short-short and long-long.

A second method of comparing results across the conditions in Figure 3 is to fit an appropriate model for the acquisition process and to examine differences in the model's parameters. For this analysis, we used an exponential function, $1-\exp (-q x)$, where $x$ is session number and $q$ is a rate parameter. ${ }^{1}$ This is an increasing, negatively accelerated curvilinear function that is equal to 0 at $x=$ 0 and has an asymptote of 1 . The model was applied to the normalized data from individual subjects, by finding the value of $q$ that maximized the variance accounted for in the first three and last five sessions of each condition. The reason for excluding the middle sessions from the parameter estimation process was that differences across conditions - especially any effect of preceding initial-link duration - were expected in only the first few postreversal sessions.

Individual and group mean parameter estimates from the model-fitting analysis are listed in Table 2. Overall, the exponential function did an excellent job of describing data from the first three and last five sessions; across subjects and conditions, the median variance accounted for was $96 \%(M=94 \%)$. When predictions for the full 20 sessions from each condition were calculated on the basis of the parameter estimates obtained with the first three and last five sessions, the model continued to describe the data well, with a median variance accounted for of $88 \%$ $(M=81 \%)$. Table 2 shows that acquisition was fastest in the short-long condition (mean $q=0.39$ ), slowest in the long-short condition (mean $q=0.16$ ), and intermediate in the short-short and long-long conditions (mean $q=$ 0.22 and 0.20 , respectively). These parameter estimates imply that the number of sessions required for preference to shift $50 \%$ of the distance to eventual asymptote was 1.79 in the short-long condition, 4.39 in the long-short condition, and 3.17 and 3.40 in the short-short and longlong conditions, respectively. An ANOVA performed on the individual parameter estimates found significant effects of preceding and following duration $[F(1,5)=20.01$ and 7.28 , respectively], with no significant interaction. 
Table 2

Best-Fitting Estimates of Rate Parameter ( $q$ ) Obtained by Fitting an Exponential Function to Normalized Preference Data From Individual Subjects

\begin{tabular}{lcccc}
\hline & \multicolumn{4}{c}{ Condition } \\
\cline { 2 - 5 } Bird & SS & SL & LS & LL \\
\hline 196 & 0.35 & 0.29 & 0.14 & 0.21 \\
197 & 0.15 & 0.28 & 0.19 & 0.16 \\
198 & 0.25 & 0.58 & 0.16 & 0.30 \\
191 & 0.16 & 0.39 & 0.19 & 0.21 \\
193 & 0.24 & 0.27 & 0.16 & 0.18 \\
194 & 0.17 & 0.52 & 0.11 & 0.16 \\
Avg. & 0.22 & 0.39 & 0.16 & 0.20 \\
$S E$ & 0.03 & 0.05 & 0.01 & 0.02
\end{tabular}

Note-SS, short-short; SL, short-long; LL, long-long; LS, long-short. See text for further explanation.

These results are consistent with the ANOVA for the data in Figure 3 reported above. They demonstrate that the rate of acquisition depends on the duration of the initial links both preceding and following the reversal.

Data from the no-food trials were further analyzed to determine whether initial-link duration had any effect on the temporal control of terminal-link responding. Responses during the no-food terminal-link trials were aggregated across the last five sessions of each condition for each subject and condition and then averaged. The results are shown in Figure 4. The filled and unfilled symbols represent the FI 16-sec and FI 8-sec terminal links, respectively. As expected, responding reached its maximum earlier on the FI $8 \mathrm{sec}$ and there was more variability in the FI 16-sec distribution, but no differences were apparent across conditions. To examine the data in more detail, repeated measures ANOVAs were conducted on median, interquartile range, and peak response rate, averaged across the last five sessions, with preceding and following initial-link duration and schedule as factors. For the medians, the effect of schedule was significant $[F(1,7)=$ 353.89], confirming that the peak occurred later on the FI 16 -sec schedule. The effects of preceding and following duration, and the interactions, were not significant. The medians, averaged across conditions and subjects, were $7.77(S E=1.17)$ for the FI $8 \mathrm{sec}$ and $14.62(S E=1.42)$ for the FI $16 \mathrm{sec}$, demonstrating that peak responding on no-food trials occurred near the schedule values. Analysis of interquartile range showed that, as expected, the effect of schedule was significant $[F(1,7)=86.67]$, with greater variability for the FI $16 \sec (M=10.40, S E=1.17)$ than for the FI $8 \mathrm{sec}(M=5.07, S E=.27)$. Unexpectedly, the effect of preceding duration was significant $[F(1,7)=$ 11.36], which suggested that interquartile range values were greater when the preceding initial-link duration was long rather than short. The effect of following duration
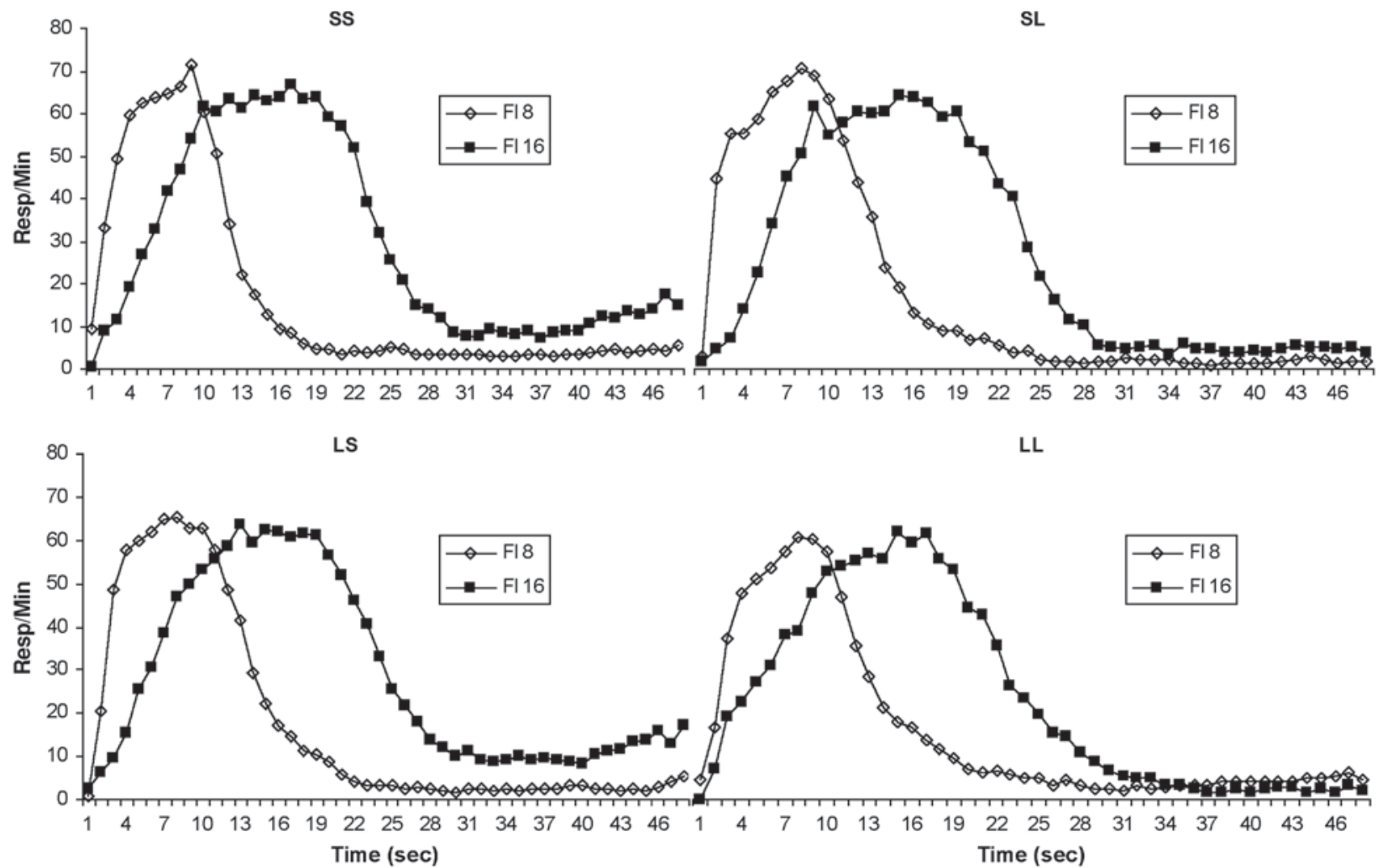

Figure 4. Average response rate (Resp/Min) over the last five sessions of each condition plotted against elapsed time (in seconds) during no-food terminal links. The unfilled diamonds represent terminal-link FI 8 sec, and the filled squares, the FI 16 sec. Data from each of the four initial-link transitions are labeled in the panels as short-short (SS), short-long (SL), long-short (LS), and long-long (LL). 
and the interactions were not significant. For peak response rate, the effect of following duration was significant $[F(1,7)=38.57]$, showing that maximum responding on peak trials was greater with short $(M=126.71$ responses/min, $S E=16.81$ ) rather than long initial links $(M=117.19$ responses $/ \mathrm{min}, S E=16.61)$. The effects of preceding duration and schedule, as well as all interactions, were not significant.

Figure 2 showed that there were no differences across conditions in asymptotic values of relative expected immediacy during no-food trials, suggesting that acquisition of temporal control did not differ across conditions (confirmed by ANOVAs on the normalized as opposed to expected immediacy data). Nevertheless, it was possible that differences in acquisition might emerge if median and interquartile range values were considered for FI $8 \mathrm{sec}$ and FI $16 \mathrm{sec}$ terminal links individually. To address this question, we conducted separate repeated measures ANOVAs for these measures from individual sessions for both schedules, with preceding duration, following dura- tion, and session as factors. For the medians, the effect of session was significant for both the FI 8-sec and FI 16 -sec terminal links $[F(19,79)=4.51$, and $F(19,79)=$ 2.81]. There were no effects of preceding and following duration, nor any significant interactions. Results were similar for interquartile range, with a significant effect of session for both FI $8 \mathrm{sec}$ and FI $16 \mathrm{sec}[F(19,79)=5.36$, and $F(19,79)=6.36]$, but no significant effects or interactions involving preceding and following duration. Thus, there were no effects of initial-link duration on median and interquartile range values for individual terminal-link schedules.

Median and interquartile range data were averaged across conditions and subjects, and results are shown in Figure 5. Clear differences in the adjustment of median and interquartile range across sessions for the FI 8 -sec and FI 16-sec terminal links are apparent. When FI $8 \mathrm{sec}$ was changed to FI $16 \mathrm{sec}$, there was a rapid overshoot in both median and interquartile range, followed by a gradual adjustment over sessions. In contrast, when FI $16 \mathrm{sec}$ was
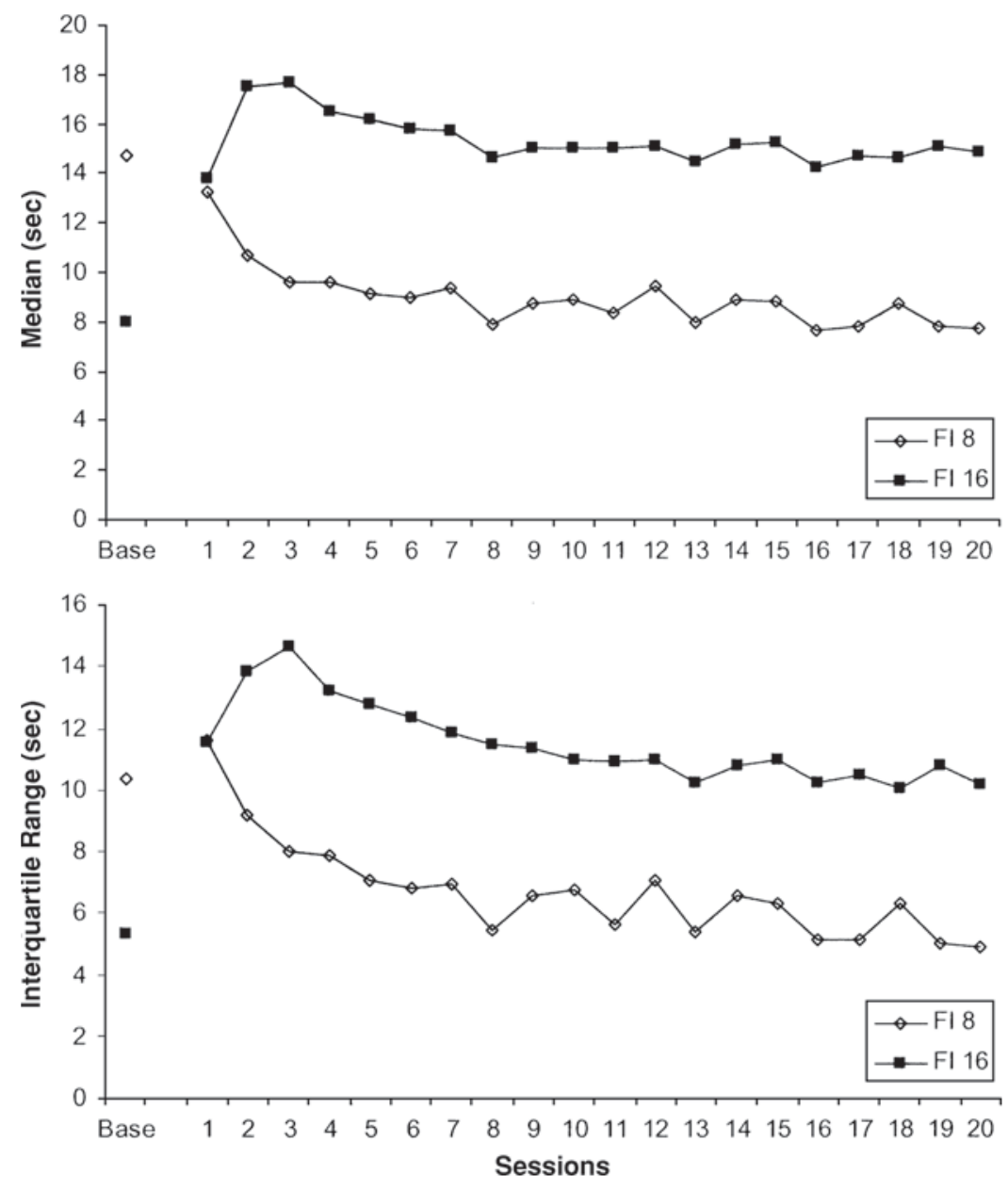

Figure 5. Average median and interquartile range (in seconds) for all subjects and conditions plotted across sessions. Base values are the average of the last five values in the previous condition. The unfilled diamonds represent the FI 8-sec terminal link, and the filled squares, the FI 16-sec. 
changed to FI $8 \mathrm{sec}$, the median and interquartile range decreased gradually (after a small overshoot for the interquartile range). These differences were confirmed through statistical analysis. A repeated measures ANOVA performed on the median data found significant effects of schedule $[F(1,5)=344.22]$ and session $[F(19,95)=$ 5.39], and a session $\times$ schedule interaction $[F(19,95)=$ 2.55]. Similarly, for interquartile range, the effects of schedule and session and their interaction were significant $[F(1,5)=64.77, F(19,95)=11.14$, and $F(19,95)=$ 2.16 , respectively].

\section{DISCUSSION}

The primary goal of this study was to investigate the effects of overall initial-link duration on acquisition of preference for unequal terminal-link FI schedules. In addition to initial-link response allocation, we were also able to examine changes in temporal control of food-reinforced behavior because $25 \%$ of terminal links were trials that extended past the FI durations ( 8 or $16 \mathrm{sec}$ ) and ended in extinction, similar to trials in the peak procedure (Roberts, 1981). The experiment used a successive-reversal design in which the location of the richer terminal link was switched every 20 sessions. Across conditions, we studied all possible combinations of initial-link duration (short/ long) both preceding and following the terminal-link reversal. The results showed that change in response allocation over the first three postreversal sessions, measured as a proportion of change to eventual asymptote, was greater when the initial links preceding the reversal were short rather than long, and when the initial links following the reversal were long rather than short. Thus, acquisition was fastest for the short-long transition (i.e., short-duration initial links preceding the reversal but long-duration initial links following the reversal), slowest for long-short, and intermediate for short-short and long-long.

These data show that the model for concurrent-chains acquisition proposed by Grace (2002a) is incomplete. Grace's model assumes that rate of acquisition depends on the pooled values of the terminal links preceding and following the reversal, computed according to a function that specifies the value of a schedule in terms of the delay distribution. Higher valued schedules (e.g., VI, short duration) produce both more interference when preceding the reversal and faster acquisition when following the reversal, in comparison with lower valued schedules (e.g., FI, long duration). Because Grace's model is based entirely on the terminal-link reinforcer delays, it is unable to predict any effects of initial-link duration.

However, these results are consistent with expectations based on a previous study by Mazur (1997). He found that individual reinforcers had greater impact on acquisition in concurrent schedules when the overall rate of reinforcement was low. If presentations of terminal-link stimuli are functionally equivalent to primary reinforcers, then Mazur's results would suggest that terminal-link stimuli should have greater impact when the overall rate of presentation is low (i.e., long initial-link duration). Note that "impact" includes both interference prior to reversal and facilitation of acquisition postreversal. It follows that acquisition should be fastest in the short-long condition, because interference is low and facilitation is high, slowest in the long-short condition (high interference, low facilitation), and intermediate in the short-short and long-long conditions. This is precisely what we observed. The similarity between our results and those of Mazur (1997) provides further support for the hypothesis that conditioned and primary reinforcers are functionally equivalent.

These effects of initial-link duration suggest that acquisition depends on the temporal context of reinforcement. Indeed, a positive relationship between initial-link duration and the effectiveness or value of a conditioned reinforcer is the core principle of Fantino's (1969) delay reduction theory (DRT) According to DRT, the effectiveness of a terminal-link stimulus as a conditioned reinforcer depends on temporal context - specifically, on the reduction in delay to reinforcement signaled by a terminal link, $T-t$, where $T$ is the overall delay to reinforcement from onset of the initial links and $t$ is the delay signaled by a terminal link. DRT correctly predicts effects of overall initial-link (Fantino, 1969) and terminal-link duration on preference in concurrent chains (MacEwen, 1972; but cf. Grace \& Bragason, 2004), and it has been extended to other situations such as foraging and observing behavior (see Fantino, 2001, for a review). For the present experiment, DRT predicts that the effectiveness or value of the terminal-link stimuli as conditioned reinforcers will be relatively strong when the initial-link schedule is VI $24 \mathrm{sec}$ and relatively weak when it is VI $8 \mathrm{sec}$. If we assume that it is easier to change the effectiveness of a relatively weak conditioned reinforcer, and that presentation of a relatively strong one produces a more rapid change in preference, then DRT would predict that acquisition should be faster in the short-long condition than in longshort. Thus, our preference acquisition data in Figure 3 are consistent with DRT.

However, the view that conditioned reinforcement value depends on temporal context is challenged by the results of Grace and Savastano (2000). In their experiments, pigeons were exposed to a two-component concurrentchains procedure (i.e., two concurrent chains in each session) in which the components differed in terms of overall initial- or terminal-link duration. After sufficient training had been provided so that initial-link preference had stabilized, terminal-link stimuli from the different components were compared in choice probe tests. Grace and Savastano found that response allocation during the probes was predicted by the reinforcer delays signaled by the stimuli in baseline. For example, in their Experiment 2, the terminallink schedules in both components were VI 10 VI $20 \mathrm{sec}$, but the initial links were either short (VI $20 \mathrm{sec}$ ) or long (VI $100 \mathrm{sec}$ ). When the VI 10-sec terminal-link stimulus from the short initial-link component was pitted against the VI $10 \mathrm{sec}$ from the long component in choice probe tests, pigeons responded equally to both alternatives. This result suggests that the pigeons had learned the delays associated with each terminal link, regardless of the dura- 
tion of the initial link. Grace and Savastano concluded that their results supported a learning-performance distinction for conditioned reinforcement: The learned value of a terminal link was determined by the scheduled reinforcer delays, while temporal context modulated the effectiveness of terminal-link stimuli as conditioned reinforcers, producing the effects of overall initial- and terminal-link duration on preference (Grace, 1994).

Some results from the present experiment are consistent with Grace and Savastano (2000). With one exception, we found no effect of initial-link duration on asymptotic peak location and variability of responding on no-food terminal links (see Figure 4). (For reasons that are unclear, there was a significant effect of initial-link duration in the preceding condition on asymptotic interquartile range in the subsequent condition.) The relative expected immediacy measure, derived from peak medians, corresponded closely to the scheduled delay ratio, indicating that subjects timed reinforcer delays accurately (see Figure 2). Also, the acquisition of temporal control in the terminal links occurred more rapidly than the corresponding acquisition of initial-link preference, which is consistent with results of Grace (2002b; see also Cerutti \& Staddon, 2004).

Although initial-link duration affected both the asymptote and rate of acquisition for initial-link response allocation, there were no corresponding effects on measures of temporal control derived from terminal-link responding. Peak location and variability of no-food response distributions were determined by the reinforcer delays signaled by the terminal links, independently of temporal context. The implication is that the reinforcing and discriminative functions of the terminal-link stimuli were dissociated, with temporal context affecting the former but not the latter. If temporal control of responding on no-food trials is interpreted as a measure of learning, then these data are consistent with the learning-performance distinction proposed by Grace (1994) for conditioned reinforcers. In any case, an important issue for future research will be to identify why initial-link duration affects the reinforcing but not discriminative function of the terminal-link stimuli. Answering this question should aid in the development of more comprehensive models that attempt to explain choice and timing through a common set of behavioral processes (see, e.g., Gallistel \& Gibbon, 2000; Gibbon, Church, Fairhurst, \& Kacelnik, 1988; but cf. Grace \& Nevin, 1999).

Figure 5 clearly shows differences in adjustment for the median and interquartile range measures between the FI 8 -sec and FI 16-sec terminal links. For the FI 8 sec to FI $16 \mathrm{sec}$ transition, change is very rapid and an overshoot was obtained, such that the median and interquartile range values increased and then decreased to an asymptotic value. Conversely, for the FI $16 \mathrm{sec}$ to FI $8 \mathrm{sec}$ transition both measures decreased gradually. Similar results to these were found with rats by Lejeune, Ferrara, Simons, and Wearden (1997), who used a peak procedure with cycled transitions to reinforcer schedule values of $10,20,30$, or
$40 \mathrm{sec}$. They found that individual peak time data jumped up rapidly or overshot when transitions were made from a lower to higher reinforcer schedule value, but peak times gradually decreased with transitions from higher to lower schedule values. Lejeune et al. noted that responding at the previously scheduled value could be extinguished on food trials when the duration was increased but not when it was decreased, and they suggested that this might account for the more rapid adjustment in the short-long transition. This explanation seems reasonable and could also apply to our data.

Overall, our results show that initial-link duration affects the rate of change of preference in concurrent chains when terminal-link schedules are reversed. Relative to eventual asymptote, change was greater when the duration of the initial links preceding the reversal was short rather than long, and when the duration of the initial links following the reversal was long rather than short. These results suggest that the impact of a conditioned reinforcer on changes in response allocation increases as a function of initial-link duration, which is consistent with DRT (Fantino, 1969). However, there were virtually no effects of initial-link duration on acquisition or asymptotic measures of temporal control during no-food terminal links. Thus, the reinforcing and discriminative functions of terminal-link stimuli were dissociated. This dissociation is consistent with an interpretation of the effects of initial-link duration on conditioned reinforcement value in terms of a learning-performance distinction (Grace, 1994; Grace \& Savastano, 2000).

\section{REFERENCES}

BAUM, W. M. (1974). Chained concurrent schedules: Reinforcement as situation transition. Journal of the Experimental Analysis of Behavior, 22, 91-101.

BAUM, W. M. (1979). Matching, undermatching, and overmatching in studies of choice. Journal of the Experimental Analysis of Behavior, 32, 269-281.

Berg, M. B., \& Grace. R. C. (2004). Independence of terminal-link entry rate and immediacy in concurrent chains. Journal of the Experimental Analysis of Behavior, 82, 235-251.

Cerutti, D. T., \& Staddon, J. E. R. (2004). Time and rate measures in choice transitions. Journal of the Experimental Analysis of Behavior, 81, 135-154.

Cheng, K., \& Roberts, W. A. (1991). Three psychophysical principles of timing in pigeons. Learning \& Motivation, 22, 112-128.

FAntino, E. (1969). Choice and rate of reinforcement. Journal of the Experimental Analysis of Behavior, 12, 723-730.

Fantino, E. (2001). Context: A central concept. Behavioural Processes, 54, 95-110.

Gallistel, C. R., \& Gibbon, J. (2000). Time, rate, and conditioning. Psychological Review, 107, 289-344.

Gibbon, J., Church, R. M., Fairhurst, S., \& Kacelnik, A. (1988). Scalar expectancy theory and choice between delayed rewards. Psychological Review, 95, 102-114.

GRACE, R. C. (1994). A contextual model of concurrent-chains choice. Journal of the Experimental Analysis of Behavior, 61, 113-129.

Grace, R. C. (1996). Choice between fixed and variable delays to reinforcement in the adjusting-delay procedure and concurrent chains. Journal of Experimental Psychology: Animal Behavior Processes, 22, 362-383.

Grace, R. C. (2002a). Acquisition of preference in concurrent chains: Comparing linear-operator and memory-representational models. 
Journal of Experimental Psychology: Animal Behavior Processes, 28, 257-276.

Grace, R. C. (2002b). The value hypothesis and acquisition of preference in concurrent chains. Animal Learning \& Behavior, 30, 21-33.

Grace, R. C., \& Bragason, O. (2004). Does the terminal-link effect depend on duration or reinforcement rate? Behavioural Processes, 67, 67-79.

Grace, R. C., \& Nevin, J. A. (1999). Timing and choice in concurrent chains. Behavioural Processes, 45, 115-127.

Grace, R. C., \& Savastano, H. I. (2000). Temporal context and conditioned reinforcement value. Journal of Experimental Psychology: General, 129, 427-443.

Lejeune, H., Ferrara, A., Simons, F., \& Wearden, J. H. (1997). Adjusting to changes in the time of reinforcement: Peak interval transitions in rats. Journal of Experimental Psychology: Animal Behavior Processes, 23, 211-231.

MacEwen, D. (1972). The effects of terminal-link fixed-interval and variable-interval schedules on responding under concurrent chained schedules. Journal of the Experimental Analysis of Behavior, 18, 253261.

MAZUR, J. E. (1997). Effects of rate of reinforcement and rate of change on choice behaviour in transition. Quarterly Journal of Experimental Psychology, 50B, 111-128.

MAzur, J. E. (2001). Hyperbolic value addition and general models of animal choice. Psychological Review, 108, 96-112.

MAZUR, J. E. (2002). Concurrent-chain performance in transition: Ef- fects of terminal-link duration and individual reinforcers. Animal Learning \& Behavior, 30, 249-260.

Mazur, J. E., Blake, N., \& McManus, C. (2001). Transitional choice behavior on concurrent-chain schedules. Behavioural Processes, 53, 171-180.

RoBerTs, S. (1981). Isolation of an internal clock. Journal of Experimental Psychology: Animal Behavior Processes, 7, 242-268.

Stubis, D. A., \& Pliskoff, S. S. (1969). Concurrent responding with fixed relative rate of reinforcement. Journal of the Experimental Analysis of Behavior, 12, 887-895.

Williams, B. A., \& DunN, R. (1991). Substitutability between conditioned and primary reinforcers in discrimination acquisition. Journal of the Experimental Analysis of Behavior, 55, 21-35.

\section{NOTE}

1. We also analyzed the data with a hyperbolic function, $y=1 /(b+x)$, where $x$ is session number and $b$ is a learning rate parameter. Although the same pattern of variation in learning rate across conditions was obtained, the hyperbolic function did not provide as good a description of the data as did the exponential; the median variance accounted for was $87 \%$ for the first three and last five sessions' data, and $78 \%$ for the full data.

(Manuscript received November 9, 2004; revision accepted for publication April 18, 2005.) 